\title{
riccafd
}

Revista Iberoamericana de Ciencias de la Actividad Física y el Deporte

\section{REVISIÓN DE INDICADORES DE RENDIMIENTO EN FÚTBOL}

\section{FOOTBALL PERFORMANCE INDICATORS REVIEW}

\author{
Reina Gómez, A.1 y Hernández Mendo, A.2 \\ 1 Doctor Ciencias Actividad Física y Deporte (F.C. Dnipro Dnipropetrovsk. Ucrania) alvaroreina@uma.es \\ ${ }_{2}$ Doctor Psicología (Universidad de Málaga. España) mendo@uma.es
}

\section{Código UNESCO: 5802.99}

Clasificación Consejo de Europa: 5

Recibido el 25 de mayo de 2012

Aceptado el 2 de julio de 2012

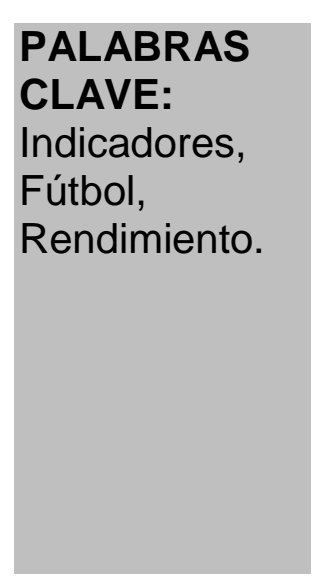

\section{RESUMEN}

Se ha realizado una revisión y selección de algunos de los factores que están implicados en el rendimiento deportivo en fútbol haciendo una recopilación de las variables que han tenido en cuenta otros investigadores y agrupándolas en diferentes bloques. En el primer bloque se presentan los factores físicos y fisiológicos, tanto los genéticos como los susceptibles de ser entrenados. En otro bloque hemos agrupado los factores contextuales en los que incluimos los psicológicos y sociales, ya que vienen a contextualizar las condiciones del partido y de los jugadores. Por último, desarrollamos los indicadores técnico tácticos más utilizados para estimar el rendimiento de los equipos de fútbol.

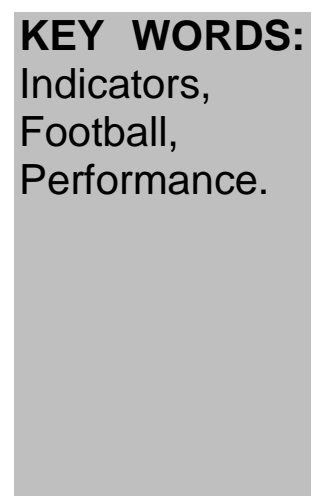

\section{ABSTRACT}

A review and selection of some of the factors that are involved in sports performance in football by a collection of variables that have been taken into account other researchers and grouped into different blocks. In the first section presents the physical and physiological factors, both genetic and capable of being trained. In another section, we have grouped the contextual factors that include the psychological and social context as they come to match conditions and players. Finally, we develop more the tactical technical indicators frequently used to estimate the performance in football teams. 


\section{INTRODUCCIÓN}

Según Lago (2008b) la varianza del resultado en diferentes partidos de fútbol surge por una parte de las diferencias sistemáticas, que son persistentes e incluyen características fundamentales y predecibles de los equipos como su planteamiento táctico, la apuesta por el dominio de la posesión del balón o no, la calidad individual de los jugadores o el tipo de defensa seleccionada. Por otra parte, surge de las diferencias no sistemáticas, que son transitorias, no podemos predecir su impacto, como los cambios producidos por alteraciones meteorológicas o los errores arbitrales.

Diferentes autores plantean el estudio de diversos indicadores 0 factores de rendimiento para dirigir el proceso de entrenamiento o para la selección de talentos. En este sentido Noa Cuadrado (2002) hace una extensa revisión de indicadores para la selección de niños con aptitudes para la práctica del fútbol.

Para hacer más sencilla su comprensión hemos agrupado en tres grandes grupos todos los factores que han sido estudiados por diferentes autores. En primer lugar se analizan los factores físicos o fisiológicos, relacionados con la actividad física desarrollada en competición (distancias, velocidades, frecuencia cardiaca...). En otro bloque se presentan los factores psicológicos y contextuales, orientados sobre el ambiente y el contexto en el que vive el jugador de fútbol, influido por los estados emocionales y la personalidad. El tercer bloque hace referencia a los aspectos específicos del fútbol, los factores técnico-tácticos y estratégicos.

\section{FACTORES FISIOLÓGICOS}

El fútbol ha sido analizado principalmente desde áreas con mayor tradición investigadora como la medicina, la fisiología o la psicología, lo que provoca que el estudio de factores de rendimiento en fútbol haya estado enfocado principalmente desde estas áreas y no desde el análisis del juego.

Durante un proceso de detección de talentos, la mayoría de los programas coinciden en que se deberían considerar factores como la herencia, el biotipo o las capacidades físicas. El análisis de la actividad física que desarrolla un jugador de fútbol resulta fundamental a la hora de determinar sus necesidades. De hecho, a medida que aumenta la experiencia del entrenador aumenta progresivamente la opinión afirmativa respecto a que los equipos cuyos jugadores realizan mayor distancia a máxima velocidad tienen más posibilidades de conseguir la victoria (Tapia, 2010). En gran cantidad de investigaciones se utilizan factores físicos y fisiológicos relacionados con el rendimiento deportivo como el registro de las distancias recorridas, la velocidad a las que se realizan éstas distinguiendo por puestos específicos, la duración de los esfuerzos, la velocidad máxima (Deutsch, Kearney y Rehrer, 2002; O'Donoghue, 2002; Reilly y Keane, 2002; Mohr, Krustrup y Bangsbo, 2003, Bachev, Marcov, Georgiev y Lliev, 2005; Zubillaga, 2006; Di Salvo, Baron, Tschan, Calderon

Rev. Ib. CE. Act.Fis. Dep. 
Montero, Bachl y Pigozzi, 2007; Randers et al., 2010). Incluso se ha estudiado en el fútbol femenino la influencia del ciclo menstrual como otro de los indicadores de rendimiento (Guijarro, De la Vega y Del Valle, 2009).

Las demandas fisiológicas del fútbol están representadas por las intensidades a las cuales se llevan a cabo las distintas actividades durante un partido. Para cuantificar la carga interna en el fútbol se han utilizado varios métodos como el registro de la frecuencia cardiaca, el lactato o el consumo de oxígeno, aunque estos últimos tienen varios inconvenientes para su uso. Las reservas de glucógeno muscular, el retardo en su agotamiento y la recuperación de dichas reservas son otros de los indicadores (Ramos Peula, 2008). El perfil antropométrico (talla, peso y porcentaje graso) también han sido identificados como variables que influyen en el rendimiento de los jugadores de fútbol.

Sin embargo, Zubillaga (2006) advierte que todas estas investigaciones tienen pocos puntos en común debido a que el tamaño de la muestra y sus características son muy diversas, la excesiva subjetividad en la determinación de las intensidades del desplazamiento de los jugadores en la acción de juego, la gran variabilidad en los protocolos y técnicas de registro, sin establecer referencias al contexto y la escasa argumentación de los niveles de validez y fiabilidad de los instrumentos utilizados. Zubillaga (2006) presenta en su tesis doctoral el resultado del análisis exhaustivo de 194 partidos de fútbol de la liga española y de competiciones europeas a través del sistema AMISCO $\AA^{\circ}$. Recoge la distancia total, la distancia en marcha, la distancia en carrera, la distancia en sprint, la distancia en alta intensidad y la frecuencia de sprint para cada uno de los jugadores y teniendo en cuenta variables como el puesto específico, la competición, los equipos, el nivel de la posesión del balón, el resultado de cada parte, el tiempo efectivo de juego, la parte del partido y el factor campo. Drust et al. (1988) indican que la posición dentro del equipo, el nivel de juego, el estilo de juego, la fatiga, y las condiciones del entorno como la temperatura, la humedad y la altitud inciden en las distancias recorridas por los jugadores. Randers et al. (2010) utilizaron varios sistemas de análisis, incluyendo el GPS y el vídeo análisis para determinar los metros recorridos teniendo en cuenta el factor de la fatiga.

\section{FACTORES PSICOLÓGICOS Y SOCIALES O CONTEXTUALES}

Existen en psicología un gran número de estudios donde se recogen distintas variables psicológicas que han resultado ser relevantes para la obtención del alto rendimiento deportivo. Estudios de los propios deportistas en forma de percepciones

comparaciones de las características psicológicas de los deportistas de éxito con otros menos afortunados mediante cuestionarios 0 tests y estudios sobre lo que piensan los entrenadores que puede ayudar a conseguir éxito en su deporte concreto. Los intentos de los psicólogos por encontrar características psicológicas 
definitorias de los deportistas más exitosos se han sucedido a lo largo de los años. Sin embargo, los resultados obtenidos no han dado lugar a conclusiones claras (Escudero, Balagué y García-Mas, 2002).

La mayoría de los programas dirigidos a mejorar el rendimiento deportivo utilizan un conjunto de técnicas para desarrollar las habilidades psicológicas de los atletas como relajación, programación de objetivos a corto y largo plazo, imaginación, control de la atención y concentración, autoinstrucciones y otras técnicas cognitivas (Gil Martínez, Capafons y Labrador, 1998; Hernández Mendo y Morales Sánchez, 2010). Desde un prisma psicológico se ha planteado la inteligencia, la creatividad, el control emocional, el estado de maduración psicofísico, la experiencia, las relaciones sociales, el medio ambiente (Capetillo, 2005), la competitividad (García Naveira y Remor, 2011). Es necesario profundizar en el conocimiento de los estados emocionales, la personalidad y la conducta específica de cada jugador, a través de una evaluación para determinar el perfil y establecer métodos y objetivos que puedan potenciar los puntos fuertes $y$ disminuir los débiles. Según Hernández Mendo y Morales Sánchez (2010) hay suficientes investigaciones que constatan la importancia de los factores psicosociales como la actitud, confianza o cohesión en el rendimiento en fútbol, pero que, sin embargo, esta importancia no queda reflejada en los planteamientos de los cuerpos técnicos a nivel profesional, abogando estos autores por la inclusión del psicólogo del deporte dentro del organigrama de los cuerpos técnicos y no tanto dentro de los cuerpos médicos.

Los aspectos psicológicos están estrechamente unidos a las condiciones sociales e institucionales del entorno del deportista, que juegan un papel importante en el desarrollo de su carrera por lo que pasan a ser también tan determinantes como los factores genéticos y fisiológicos. Cada atleta es un individuo con necesidades y preferencias únicas, teniendo mayores posibilidades de éxito en la competición de alto rendimiento cuanto más satisfechos están (Graham y Cross, 2005). Los equipos con una mayor cohesión de equipo y una actitud positiva, moral y ánimo, tienen más probabilidad de conseguir la victoria (Tapia, 2010). De este modo, los estados de ánimo previos a la competición, influyen en el rendimiento de un equipo de fútbol durante toda la temporada y esta relación está mediada por la variable localización del partido, siendo los partidos jugados fuera de casa donde más se manifiestan los estados de ánimo y su consiguiente influencia en el rendimiento (Sánchez Pérez, 2001).

Del mismo modo, los factores sociales y psicológicos los podemos interpretar como factores contextuales del partido. Los estados de ánimo de cada jugador, la meteorología (Tapia, 2010), la alimentación e hidratación, el estado del terreno de juego, el factor local vs visitante, el árbitro, adelantarse en el marcador (Castellano, 2008a; Tapia, 2010) o

Rev. Ib. Cᄃ. Act.Fis. Dep. 
el rival, son variables que van a contextualizar el encuentro. Reina (2012) estudia precisamente el rival, el árbitro, el factor campo, el puesto específico y los jugadores alineados para estimar su influencia en el resultado del partido.

Tapia (2010) advierte que a medida que aumenta la titulación académica aumenta la opinión de que el planteamiento del partido se debe realizar teniendo en cuenta las características del equipo contrario pero en cambio, el $30,1 \%$ de los entrenadores andaluces opina que el planteamiento del partido no lo realizan teniendo en cuenta el rival, lo realizan teniendo en cuenta las ocasiones de gol en contra, el resultado, el seguimiento del plan o modelo táctico previsto y jugar en superioridad 0 inferioridad numérica. Sin embargo, los datos aportados por Reina (2012) confirman la influencia del rival en el resultado del partido. Por otra parte, el $42,2 \%$ de los entrenadores de fútbol andaluces opina que las características y el estado del terreno de juego es uno de los factores más importantes.

La ventaja de jugar en casa en los diferentes deportes ha sido un objeto de estudio ampliamente abordado en los últimos veinte años en la literatura sobre psicología del deporte (Bray y Widmeyer, 2000; Carron, Loughhead y Bray, 2005; Voyer, Kinch y Wright, 2006; Pollard, 2006, 2008; Lago y Martín Acero, 2007; Marcelino, Mesquita y Afonso, 2008; Lago, 2009; Reina 2012), pudiendo aumentar la probabilidad de ganar el partido (Tapia, 2010). Otras investigaciones (Clarke y Norman, 1995; Nevill,
Newell y Gale, 1996; Pollard, 2002; Nevill, Balmer y Williams 2002; Lucey y Power, 2004; Carmichel y Thomas, 2005; Jacklin, 2005; Pollard y Pollard, 2005; Boyko et al., 2007; Balmer et al., 2007; Montes y Sala, 2009; Taylor, Mellalieu, James, Barter, 2010) estudian la ventaja de jugar en campo propio y explican el mayor numero de victorias de los equipos locales como consecuencia de su mayor efectividad, entrega y del apoyo de los aficionados con independencia del comportamiento arbitral. Reina (2012) obtiene significativamente un mayor número de lanzamientos a puerta en casa y tendente a la significación un mayor número de acciones de 1 contra 1, ambas acciones altamente correlacionadas con los goles a favor. Estos datos concuerdan con Lago y Lago (2011) que concluyen que los equipos locales tienen mayor frecuencia de los indicadores de ataque pero esta ventaja es distinta en función del nivel del equipo.

Es reconocido por entrenadores, jugadores y aficionados que a veces el azar es unos de los factores que influyen en el resultado de un partido (Lago, 2005). Esta opinión disminuye progresivamente conforme aumenta la titulación del entrenador (Tapia, 2010). Sin embargo, la importancia del azar puede ser importante para un único partido o una serie muy pequeña, pero a partir de cierto número de partidos, la suerte no es un factor determinante (Lago, 2005). Algunos autores se han referido a este factor en sus investigaciones (Álvaro, 2005; Lago, 2005, 2007, 2008a).

Rev. Ib. CE. Act.Fis. Dep. 
Las actuaciones de los árbitros están sometidas a un exhaustivo análisis y a todo tipo de críticas por parte de los medios de comunicación, los aficionados, los jugadores y entrenadores. El tiempo extra añadido al final del partido, los fueras de juego, las faltas y las tarjetas mostradas pueden influir en el resultado final. Por ese motivo, el árbitro es considerado como otro factor que influye en el rendimiento en fútbol profesional tal y como advierte Reina (2012), que obtiene un alto porcentaje de la variancia asociada a la variable (faceta) árbitro.

Según Garicano, Palacios-Huertas y Prendergast (2005) los árbitros favorecen al equipo local añadiendo más tiempo si el resultado es ajustado y adverso para el equipo local o reduciéndolo en caso contrario. Sutter y Kocher (2004) estudian el comportamiento de los árbitros alemanes con respecto al tiempo extra y a los penaltis señalados, confirmando la existencia de un favoritismo sistemático hacia el equipo local. La presión que los aficionados locales ejercen sobre el árbitro puede influir sobre sus decisiones como se analiza en las investigaciones de Dohmen $(2003,2008)$ y PetterssonLidbom y Priks (2007). Del mismo modo, Reina (2012) obtiene un alto porcentaje de la variancia asociada a la interacción entre el árbitro del partido y el campo donde se juega.

Montes y Sala (2009) analizan los resultados de las temporadas 20022003 a 2007-2008 de la Liga Española de Primera División con el fin de determinar la influencia de los árbitros tanto desde el punto de vista del tiempo extra que añaden como de las sanciones disciplinarias (faltas y tarjetas) teniendo en cuenta la posible influencia de la presión ambiental. Se concluye que no puede afirmarse que exista influencia arbitral de forma generalizada, aunque algunos árbitros en concreto sí parecen influir en los resultados, contrastando el escaso valor del tiempo extra para cambiar el resultado de un partido.

Otros aspectos que proponemos a tener en cuenta como factores que pueden influir en el rendimiento son el hecho de que previo o a posteriori del partido de competición haya habido concentración de selecciones nacionales y los jugadores hayan competido con sus países, con un determinado desplazamiento que podríamos contabilizar. Igualmente, que el propio equipo compita entre semana por competiciones europeas, copa del rey o por jornada de liga también debería ser una variable a estudiar.

\section{FACTORES TÉCNICO-TÁCTICOS Y ESTRATÉGICOS}

Los iniciales modelos de análisis del rendimiento que se extendieron en los deportes de equipo fueron extraídos de deportes individuales. En estas especialidades los factores técnicos y biomecánicos son determinantes. Sin embargo, estos modelos aplicados en los deportes colectivos no permiten comprender la lógica interna del juego mientras los indicadores tácticos sí lo permiten. Los indicadores técnicos y biomecánicos son una expresión exclusiva del rendimiento individual, que no del colectivo, que quizás

Rev. lb. C.. Act.Fis. Dep. 
permitan entender mejor al jugador pero no al juego.

Una primera aproximación investigadora, considera como factor de pertinencia e indicador de máxima eficacia, la consecución del gol. Analizan los eventos precedentes a la consecución del gol, teniendo en cuenta variables como el número de pases, el número de jugadores que intervienen, la zona de lanzamiento, el momento temporal, la duración total, el tipo de inicio de jugada, el nivel de la oposición, la superficie de contacto, entre otros (Gréhaigne, 2001; Mas, 2003; Ensum, Pollard y Taylor, 2005; Hughes y Churchill, 2005; Abt, Dickson y Mummery, 2002; Fernández-García, et al. 2003; Reina, 2006; Reina, Hernández Mendo y FernándezGarcía, 2010).

En los deportes de equipo de tanteo alto (baloncesto, balonmano) la selección de los indicadores del rendimiento que dan cuenta del juego y del resultado parece sencilla. Al tratarse de deportes de finalización, donde cada jugada termina con un lanzamiento o una acción próxima a la meta rival (canasta o portería), el resultado final es la consecuencia del éxito en cada una de las posesiones o unidades de competición de cada equipo. Las causas que conducen al éxito o fracaso son cuantificables $y$ tienen un efecto claro en el marcador parcial de cada posesión y final (Alvaro et al., 1995; Trninié, Milanovic y Dizdar, 1997). Sin embargo, en los deportes de tanteo bajo (fútbol, hockey hierba) la propuesta de los indicadores del rendimiento que contienen el

Rev. Ib. CE. Act.Fis. Dep. desarrollo del juego es mucho más difícil. La finalización, independientemente de su consecuencia, es en sí mismo un indicador de éxito dentro del juego. La identificación de las causas que condujeron a la evolución del partido y al resultado final resulta muy compleja. A veces, una causa pequeñísima (una decisión arbitral, un cambio en la alineación) provoca un efecto considerable en el juego; $y$ a veces una causa aparentemente enorme (una expulsión, un gol) no tiene consecuencias perceptibles en el juego (Lago, 2008a). Por este motivo, las medidas más amplias de la efectividad ofensiva, tales como oportunidades de gol y tiros a gol, se usan comúnmente como alternativa a los goles marcados debido a la baja probabilidad de dar un resultado natural (cerca de 1\%) en el fútbol (Tenga, Ronglan y Bahr, 2010).

Partiendo de esto, en diferentes investigaciones se consideran indicadores como el número de chut a puerta (Hughes; Robertson y Nicholson, 1988; Tapia et al. 2005; Castellano, Perea y Hernández Mendo, 2008; Tapia, 2010), el número de centros y remates (Molina, 2006, López, Vegas, Martín y Casimiro, 1998), entre otros. De Bortoli, De Bortoli y Márquez (2001) realizaron un seguimiento de los lanzamientos teniendo en cuenta los lanzamientos fuera de portería, los lanzamientos a meta y los lanzamientos que acababan en gol, verificando si existe alguna relación entre éstos y el rendimiento en el campo estimado en función del resultado final de los partidos. A través de estos parámetros se obtienen diversos índices y llegan a 
la conclusión que la cualidad de los lanzamientos tiene mayor importancia que su cantidad. En la misma línea Lago et al. (2010) realizan un análisis secuencial retrospectivo de las acciones que preceden al lanzamiento a portería y consideran dichas acciones como indicadores de éxito.

López Thaureaux y Noa Cuadro (2003) hablan de varios indicadores como la relación entre goles marcados y el número de ataques a favor; la relación entre goles marcados, tiros a favor y ocasiones de gol; la relación entre goles marcados y los tiros a favor; la relación entre los goles permitidos y acciones defensivas realizadas; la relación entre tiros permitidos y acciones defensivas ejecutadas; y la relación entre goles permitidos y tiros realizados por el equipo adversario. En otra línea, Valez, Areces, Blanco y Arce (2011) diseñaron una batería de indicadores (iniciativa de juego, carga física, volumen de juego ofensivo, precisión de juego ofensivo y progresión en el juego ofensivo) para evaluar el rendimiento.

Una segunda aproximación al estudio de los parámetros tácticos y estratégicos incide en la posesión del balón como parámetro relevante y de alta significación estratégica en la acción de juego en el fútbol. No obstante, hasta hace unos años, uno de los hallazgos más aceptados era que la posesión del balón no tenía demasiado que ver con el resultado $\mathrm{y} / \mathrm{o}$ el rendimiento de los equipos en la competición (Dawson, Dobson y Gerrard, 2000; Hadley, Poitras, Ruggiero y Knowles, 2000;
Carmichael, Thomas y Ward, 2001; Gómez y Álvaro, 2002), o su relación era poco evidente (Hughes y Bartlett, 2002; James, Jones y Mellalieu, 2004). Sin embargo, Lago (2008a) comenta que esta inferencia realizada por los investigadores puede venir por problemas metodológicos.

Existen diferentes investigaciones que relacionan el rendimiento y la posesión de balón de los equipos (Carmichael, Thomas y Ward, 2001; Gómez y Álvaro, 2002; Lago, Martín Acero, Seirul-lo y Álvaro 2006, Castellano, 2008b). Según Tenga et al. (2010) los contraataques son más eficaces que los ataques elaborados, no teniendo más probabilidad de conseguir gol con las jugadas de larga posesión de balón, aunque el $65,3 \%$ de los entrenadores andaluces encuestados opinan que el equipo que tiene mayor porcentaje de posesión de balón tiene más posibilidades de conseguir la victoria (Tapia, 2010). Con relación a los factores que determinan el tiempo de posesión Lago, Martín Acero y Seirul-lo (2007) desarrollan un modelo de regresión lineal que maneja como variables independientes los minutos que los equipos tienen el marcador en contra, a favor o empatado a lo largo de cada partido, tres variables que identifican a cada uno de los equipos y sus rivales y el carácter local o visitante. Llegan a la conclusión que los equipos locales tienen la posesión del balón prácticamente 6 puntos porcentuales más que los visitantes. Cada 10 minutos con el marcador en contra incrementa en casi 1 punto el tiempo de posesión del

Rev. lb. CL. Act.Fis. Dep. 
balón, mientras que cada 10 minutos con el marcador empatado supone sumar para el equipo local 0,45 unidades porcentuales más de dominio del balón. En este mismo sentido, James, Jones y Mellalieu (2004) sugieren que la posesión del balón es mayor para los equipos que terminan ganando, cuando van todavía perdiendo o empatando, que para los perdedores, pero que no hay diferencias cuando los equipos ganadores ya dominan en el marcador (Lago, Martín Acero y Seirul-lo, 2007).

Otra perspectiva estudia los movimientos que realizan los jugadores a partir del análisis de los desplazamientos de los jugadores involucrados en la acción de juego (Grehaigne, Bouthier y David, 1997), las relaciones entre jugadores 0 asociaciones que se establecen en un equipo de fútbol durante la actividad competitiva (Castellano, Hernández Mendo y Haro, 2002).

En otra línea de investigación, algunos autores tratan de analizar las acciones que se inician a balón parado, estudiando su incidencia en el juego y el marcador (Sousa y Garganta, 2001; Beas Martínez, 2007, Acar et al., 2009; Reina, 2012). Existe una asociación significativa entre el nivel de competición entrenado y la opinión que con jugadas de estrategia hay mayor probabilidad de conseguir gol aunque no está reforzado mayoritariamente por los entrenadores con estudios universitarios y de tercer ciclo (Tapia, 2010).
Se han revisado diversos trabajos como el estudio de Hughes et al (1988), que registra variables como faltas, regates, carreras, despejes, pases, pérdidas de posesión, saques de banda, saques de falta, saques de esquina, remates a puerta, golpeos con la cabeza y centros. En cada registro se recoge el tiempo, la zona donde tiene lugar, el jugador que la realiza y la acción, encontrando diferencias significativas en varios de los elementos analizados. Casáis, Lago, Lago, Iglesias y Gómez (2011) registran tiros totales, tiros dentro, eficacia, asistencias, centros, fueras de juego incurridos, faltas recibidas, corners a favor, posesión de balón, centros recibidos, fueras de juego forzados, faltas cometidas, corners recibidos, tarjetas amarillas, tarjetas rojas $y$ localización del partido para diferenciar los equipos ganadores de los perdedores. Igualmente Reina (2012) estudia este mismo tipo de acciones técnicas pero añade las consecuencias de dichas acciones como provocar estrategia a favor 0 en contra, recibir o provocar falta y recibir o provocar tarjeta. Por otra parte, Gerisch y Reichelt (1993) analizan las situaciones de 1 contra 1 , registrando el tiempo, el jugador, la acción de juego, la zona del espacio de juego, el resultado (ganado o perdido) y el jugador oponente, estableciendo la existencia de relación entre el éxito en las situaciones de 1 contra 1 y la victoria en el partido, resultados que concuerdan con los de Reina (2012) que obtiene una alta correlación entre las acciones de 1 contra 1 y los goles a favor.

Rev. Ib. Cᄃ. Act.Fis. Dep. 
Por último, una cuestión que debe ser considerada es que no todos lo equipos tienen el mismo modelo de juego ni pretenden dominar los mismos aspectos del partido. Esta característica determina que la selección de los indicadores a utilizar para valorar el rendimiento de los equipos deba ser específica e individual para cada equipo. No pueden ser utilizados los mismos indicadores del rendimiento en dos equipos que tienen diferentes objetivos de juego (Lago y Martín Acero, 2005). En definitiva, los mismos indicadores del rendimiento pueden tener una potencia explicativa muy diferente para dos equipos distintos o para un mismo conjunto en dos momentos de la competición.

\section{REFERENCIAS BIBLIOGRÁFICAS}

1. Abt, GA, Dickson, G y Mummery, WK. (2002). Goal scoring patterns over the course of a match: an analysis of the Australian national soccer league. En Spinks, W., Reilly, T. y Murphy, A. (Eds.). Science and Football IV Londres: Routledge; 2002. p. 106-111.

2. Acar, M.F., Yapicioglu, B., Arikan, N., Yalcin, N., Ates, N. y Ergun, M. (2009). Analysis of goals scored in the 2006 World Cup. En T. Reilly and A. F. Korkusuz (Ed.), Science and Football VI London and New York: Routledge; 2009. p. 235-242.

3. Alvaro, J., Dorado, A., González Badillo, J.J., González, J.L., Navarro, F., Molina, J.J., Portoles, J. y Sánchez, F. Modelo de análisis de los deportes colectivos basado en el rendimiento en competición. INFOCOES 1995;1: 2140.

4. Bachev, V., Marcov, P., Georgiev, P. y Lliev, M. (2005). Analyses of Intensity of Physical Load during a Soccer Match. En T. Reilly, J. Cabri y D. Araujo, D. (Eds.) Science and Football V Londres: Routledge; 2005. p. 231236.

5. Balmer, N., Nevill, A., Lane, A., Ward, P., Williams, M. y Fairclough, $S$.
Influence of crowd noise on soccer refereeing consistency in soccer. Journal of Sport Behavior 2007;30(2):130-145.

6. Beas Martínez, M.A. Obervación y análisis de las acciones a balón parado en el fútbol profesional. [Diploma de Estudios Avanzados]. Universidad de Málaga;2007.

7. Boyko, R.J., Boyko, A.R. y Boyko, M.G. Referee bias contributes to home advantage in English Premiership football. Journal of Sports Sciences 2007;25 (11):1185-1194.

8. Bray, S.R. y Widmeyer, W.N. Athlete's perceptions of the home advantage: an investigation of perceived causal factors. Journal of Sports Behavior 2000;23 (1):1-10.

9. Capetillo, R. Factores condicionantes de la producción de rendimiento del futbolista. Lecturas: educación física y deportes. Revista Digital. 2005. 10, (91). Disponible en: http://www.efdeportes.com/efd91/rendi m.htm.

10. Carmichael, F. y Thomas, D. HomeField Effect and Team Performance. Journal of sports economics 2005;6(3):264-281.

11. Carmichael, F., Thomas, D. y Ward, R. Production and Efficiency in Association Football. Journal of Sports Economics 2001;2(3):228-243.

12. Carmichael, F., Thomas, D. y Ward, R. Production and Efficiency in Association Football. Journal of Sports Economics 2001;2(3):228-243.

13. Carron, A., Loughhead, T. y Bray, S. The Home Advantage in Sport Competitions: Courneya and Carron's (1992). Conceptual Framework a Decade Later. Journal of Sports Sciences 2005;223(4):395-407.

14. Casáis, L, Lago, C., Lago, J., Iglesias, S. y Gómez, M. (2011). Indicadores de rendimiento competitivo que diferencias equipos ganadores y perdedores de la liga española. Revista de preparación física en el fútbol 2011;2:44-53.

15. Castellano, J. En Pasado, presente y futuro del fútbol. En J. Castellano (ed.), Fútbol e innovación. Sevilla: Wanceulen; 2008ª. p. 37-67.

16. Castellano, J. (b). Análisis de las posesiones de balón en fútbol: frecuencia, duración y transición.

Rev. Ib. Cᄃ. Act.Fis. Dep. 
Motricidad. European Journal of Human Movement 2008;21:189-207.

17. Castellano, J., Hernández Mendo, A. y Haro, J.A. Mapas socioconductuales de la selección francesa en el mundial de fútbol de francia'98. Psicología del Deporte 2002;11(1):35-51.

18. Castellano, J., Perea, A., y Hernández Mendo, A. Análisis de la evolución del fútbol a lo largo de los mundiales. Psicothema 2008;20(4):929-932.

19. Clarke, S.R. y Norman, J.M. Home ground advantaje of individual clubs in English soccer. Statistician 1995;44:509-521.

20. Dawson, P., Dobson, S. y Gerrard, B. Stochastic Frontiers and the Temporal Structure of Managerial Efficiency in English Soccer. Journal of Sports Economics 2000;4(1):24-32.

21. De Bortoli, A.L., De Bortoli, R. y Márquez, S. Utilización de coeficientes ofensivos para el análisis del rendimiento deportivo en el fútbol sala. Motricidad: Revista de Ciencias de la Actividad Física y del Deporte 2001;7:7-17.

22. Deutsch, M.U., Kearney, G.A. y Rehrer, N. J. A Compararison of competition work rates in elite club and "Super 12" rugby. En Spinks, W., Reilly, T. y Murphy, A. (Eds.). Science and Football IV Londres: Routledge; 2002. p. 160166.

23. Di Salvo, V., Baron, R., Tschan, H., Calderon Montero, F.J., Bachl, N. y Pigozzi, F. (2007). Performance Characteristics According to Playing Position in Elite Soccer. International Journal of Sports Medicine 2007;28(3):222-227.

24. Dohmen, T.J. In support of the supporters? Do social forces shape decisions of the impartial? IZA Discussion paper 2003; 755.

25. Dohmen, T.J. The influence of social forces: evidence from the behaviour of football referees. Economic Inquiry, 2008;46(3):411-424.

26. Drust, B., Reilly, T. y Rienzi, E. Analysis of work rate in soccer. Sports exercice and injury 1988;IV(4):151-155.

27. Ensum, J., Pollard, R. y Taylor, S. Applications of logistic regression to shots at goal in association football. En T. Reilly, J. Cabri y D. Araujo, D. (Eds.) Science and Football V. Londres: Routledge; 2005. p. 211-218.

Rev. Ib. Cᄃ. Act.Fis. Dep.
28. Escudero, J.T., Balagué, G. y GarcíaMas, A. Comportamientos que influyen en el rendimiento deportivo de jugadores de baloncesto desde el punto de vista de los entrenadores. Psicothema 2002;14(1):34-38.

29. Fernández-García, J.C., Reina, A., Ruiz Aparicio, J.A., Martín Recio, F.J. y Ruiz de Alarcón, A. (2003). Análisis observacional del gol y el tiempo de juego en fútbol-7. En II Congreso Mundial de Ciencias de la Actividad Física y el Deporte. Deporte y Calidad de Vida. 2003. Granada ; p. 70-74.

30. García Naveira, A. y Remor, E. Motivación de logro, indicadores de competitividad y rendimiento en un equipo de jugadores de fútbol de competición varones entre 14 y 24 años. Universitas Psychologica, 2011;10(2):477-487.

31. Garicano, L., Palacios-Huertas, I. y Prendergast C. Favoritism under socialpressure. The Review of Economics and Statistics 2005;87:208216.

32. Gerisch, G. y Reichelt, M. (1993). Computer- and video-aided analysis of football games. En T. Reilly, J. Clarys y A. Stibbe (eds), Science and Football II. London: E. and F. N. Spon; 1993. p. 167-173.

33. Gil Martínez, J., Capafons, A. y Labrador, F. Programa psicológico para mejorar los resultados de jugadores de balonmano. Psicothema 1998;10:271280.

34. Gómez López, M. y Álvaro, J. El tiempo de posesión como variable no determinante del resultado en los partidos de fútbol. El Entrenador Español 2003;97:39-57.

35. Graham, E. Cross, N. (2005). Entrenamiento para el Rendimiento: Individualización de los Programas de Entrenamiento. G-SE Standard. [citado 20 marzo 2011] Disponible en: http://www.g-se.com/pid/477.

36. Gréhaigne, J. F., Bouthier, D. y David, B. (1997). A method to analyse attacking moves in soccer. En T. Reilly, J. Bangsbo and M. Hughes (eds.), Science and Football III London: E. and F. N. Spon. 1997; p. 258-266.

37. Gréhaigne, J.F. Time distribution of goals in soccer: some championships and the 1998 World Cup. En Hughes, M. y Tavares, F. (Eds.). Proceedings of 
the IV Congress of Notational Analysis of Sport. Oporto; 2001. p. 41-50.

38. Guijarro, E., De la Vega, R. y Del Valle, S. Ciclo menstrual, rendimiento y percepción del esfuerzo en jugadoras de fútbol de élite. Revista Internacional de Medicina y Ciencias de la Actividad Física y el Deporte 2009;9(34):96-104.

39. Hadley, L., Poitras, M., Ruggiero, J. y Knowles, S (2000). Performance Evaluation of National Football League Teams, Managerial and Decision. Economics 2000;4(21):45-56.

40. Hernández Mendo, A. y Morales Sánchez, V. (2010). Psicología y fútbol profesional: caracterización de un reto pendiente. Apuntes de Psicología 2010;28(2):237-262.

41. Hughes, M. y Churchill, S. Attacking profiles of successful and unsuccessful teams in Copa America 2001. En T. Reilly, J. Cabri y D. Araújo (Eds.), Science and Football V. Londres: Routledge; 2005. p. 219-224).

42. Hughes, M., Robertson, K. y Nicholson, A. (1988). Comparison of patterns of play of successful and unsuccessful teams in the 1986 World Cup for soccer. En T. Reilly, Lees, K. Davis y W.J. Murphy (Eds.), Science and Football I Londres: E. y F.N.; 1988. p. 363-367.

43. Hughes, M., Robertson, K. y Nicholson, A. (1988). Comparison of patterns of play of successful and unsuccessful teams in the 1986 World Cup for soccer. En T. Reilly, Lees, K. Davis y W.J. Murphy (Eds.), Science and Football I Londres: E. y F.N.; 1988. p. 363-367.

44. Hughes, M.D. y Bartlett, R. The use of performance indicators in performance analysis. Journal of Sports Sciences, Special Edition 2002;20:739-754.

45. Jacklin, P.B. Temporal changes in home advantage in English football since Second World War: what explains improved away performance? Journal of Sports Sciences 2005;23:669-679.

46. James, N., Jones, P.D. y Mellalieu, S.D. Possession as a Performance Indicator in Soccer. International Journal of Performance Analysis in Sport 2004;4(1):98-102.

47. Lago, C. Ganar o perder en el fútbol de alto nivel. ¿Una cuestión de suerte? Motricidad. European Journal of Human Movement 2005;14:137-152.

Rev. Ib. CL. Act.Fis. Dep.
48. Lago, C. ¿Por qué no pueden ganar la liga los equipos modestos? La influencia del formato de competición, sobre el perfil de los equipos ganadores. Motricidad. European Journal of Human Movement 2007;18:135-151.

49. Lago, C. El análisis del rendimiento en el fútbol. Estado actual y perspectivas de futuro de la investigación. En Castellano, J. (2008). Fútbol e innovación. Sevilla: Wanceulen; 2008ª p. 89-103.

50. Lago, C. El análisis del rendimiento en los deportes de equipo. Algunas consideraciones metodológicas. Asociación Científico Cultural en Actividad Física y Deporte (ACCAFIDE). Las Palmas de Gran Canaria.2008b; Revista no 1 EneroJunio.

51. Lago, C. The influence of match location, quality of opposition, and match status on possession strategies in professional association football. Journal of Sports Sciences 2009;27(13):1463.

52. Lago, C. y Lago, J. Game location and team quality effects on performance profiles in professional soccer. Journal of Sports Science and Medicine 2011;10:465-471.

53. Lago, C. y Martín Acero, R. Análisis de variables determinantes en el fútbol de alto rendimiento: el tiempo de posesión del balón (abriendo la caja negra del fútbol). Revista de Entrenamiento Deportivo 2005; XIX(2):13-20.

54. Lago, C. y Martin Acero, R. Determinants of possession of the ball in soccer. Journal of Sports Sciences 2007;25(9):969-974.

55. Lago, C., Cancela, JM, Fernández Braga, F., López Graña, MP, Veiga, J. Evaluación de las acciones ofensivas en el fútbol de rendimiento mediante indicadores de éxito en diseños diacrónicos intensivos retrospectivos. Apunts 2010;72:96-102.

56. Lago, C., Martín Acero, R. y Seirul-lo, F. (2007). El rendimiento en el fútbol. Una modelización de las variables determinantes para el F.C. Barcelona, Apunts 2007;90:51-58.

57. López Thaureaux y Noa Cuadrado, $\mathrm{H}$. Propuesta de indicadores para valorar el rendimeinto técnico-táctico de un 
equipo de fútbol. Revista Facultad de Cultura Física Holguín,2003;1(9):1-8.

58. López, R. Vegas, G, Martín, J.O. y Casimiro, F.M. Actuación defensiva ante oponente directo sin balón en pases laterales. III Simposium de investigación en el fútbol. 1988; Sevilla.

59. Lucey, B. y Power, D. Do soccer referees display home team favouritism? 2004; Woking paper Trinity College Dublin.

60. Marcelino, R., Mesquita, I. y Afonso, J. (2008). The weight of terminal actions in Volleyball. Contributions of the spike, serve and block for the teams rankings in the World League 2005. International Journal of Performance Analysis in Sport 2008;88(2):1-7.

61. Mas, J. Análisis descriptivo del microsistema colaboración oposición de los jugadores próximos al balón a través de un estudio observacional de la circulación del balón en fútbol de alto nivel: posibles incidencias sobre la táctica y estrategia del juego. .[Tesis Doctoral] Universidad de Granada; 2003

62. Mohr, M. Krustrup, P. y Bangsbo, J. Match performance of high-standard soccer players with special reference to development of fatigue. Journal of sport Sciences 2003;21:519-528.

63. Molina, F.J. Fútbol: Recursos y consignas para el entrenamiento técnico-táctico (Barcelona): Paidotribo; 2006

64. Montes, F. y Sala, R. ¿Tienen los árbitros de fútbol influencia en los resultados de la liga española? XVII Jornadas ASEPUMA - V Encuentro Internacional. 2009; 17 (1), p. 102.

65. Nevill, A.M., Balmer, N.J. y Williams, A.M. The influence of crowd noise and experience upon refereeing decisions in association football. Psychology of Sport and Exercise 2002;3:261-272.

66. Nevill, A.M., Newell S. y Gale, S. Factors associated with home advantage in English and Scottish Soccer. Journal of Sports Sciences 1996;14:181-186.

67. Noa Cuadrado, H. Un nuevo enfoque sobre los criterios de selección en el fútbol. Revista Digital de Educación Física y Deportes. 2002 [citado 16 abril 2010] 8, 48 Disponible en: http://www.efdeportes.com/efd104/sele ccion-de-talentos.htm.

Rev. lb. Cᄃ. Act.Fis. Dep.
68. O'Donoghue P.G. Time-motion analysis of work-rate in English FA Premier League soccer. International Journal of Performance Analysis in Sport 2002;2(8):36-43.

69. Pettersson-Lidbom, P. y Priks, M. Behavior under social pressure: Empty Italian stadiums and referee bias. (Munich):CESifo Working Paper Series; 2007.

70. Pollard, R. Evidence of a reduced home advantage when a team moves a new stadium. Journal of Sports Sciences 2002;20:969-973.

71. Pollard, R. Worldwide regional variations in home advantage in association football. Journal of Sports Sciences 2006;24(3):231-240.

72. Pollard, R. Home advantage in football: A current review of an unsolved puzzle. The Open Sports Sciences Journal 2008;11:12-14.

73. Pollard, R. y Pollard, G. Long-term trends in home advantage in professional team sports in North America and England (1876-2003). Journal of Sports Sciences 2005;23(4):337-350.

74. Ramos Peula, LE. (2008). Implicación del glucógeno muscular sobre el rendimiento en el fútbol profesional. Revista Digital de Educación Física y Deportes. 2008 [citado 25 octubre 2009]; 13 (124) Disponible en: http://www.efdeportes.com/efd124/impli cacion-del-glucogeno-muscular-sobreel-rendimiento-en-el-futbolprofesional.htm.

75. Randers, M.B., Mujika, I., Hewitt, A., Santisteban, J., Bischoff, R., Solano, R., Zubillaga, A., Peltola, E., Krustrup, P. y Mohr, M. Application of four different

football match analysis systems: A comparative study. Journal of Sports Sciences 2010;28(2):171.

76. Reilly, T. y Keane, S. Estimation of physiological strain on Gaelic football players during match-play. En Spinks, W., Reilly, T. y Murphy, A. (Eds.) Science and Football IV Londres: Routledge.. 2002;p. 157-166.

77. Reina, A. Influencia del feedback audiovisual en la iniciación deportiva. Observación y análisis del gol en fútbol-7. [Diploma de Estudios Avanzados]. Universidad de Málaga; 2006 
78. Reina, A. Evaluación de factores implicados en el rendimiento del fútbol profesional. [Tesis Doctoral] Universidad de Málaga; 2012

79. Reina, A., Hernández Mendo, A. y Fernández-García, J.C. Multi-facet design for goal scoring in soccer-7. Quality y Quantity 2010;44 (5):10251035.

80. Sánchez Pérez, A. Estados de ánimo y rendimiento deportivo en fútbol: ¿existe la ventaja de jugar en casa? Revista de psicología del deporte 2001;10 (2):197210.

81. Sousa, T. y Garganta, J. (2001).The importance of set-plays in soccer. En Hughes, M. y Tavares, F. (Eds.). Proceedings of the IV Congress of Notational Analysis of Sport. (pp.5357). Oporto: Centre for Team Sports Studies.

82. Sutter, M. y Kocher M.G. Favoritism of agents. The case of referees' home bias. Journal of Economic Psicology 2004;25:461-469.

83. Tapia, A. El entrenador en la dirección de equipos de fútbol y factores determinantes en el resultado del partido. [Tesis Doctoral] Universidad de Málaga; 2010.

84. Tapia, A., Reina, A., Díaz Martínez, F., Berrocal, M.A., García Navarro, J.A., Gálvez, P. y Hernández Mendo, A. (2005). Observación de las acciones ofensivas en un equipo de fútbol. Lecturas: Educación Física y Deportes. Revista Digital. 2005 [citado 2 marzo 2007]; 12(108). Disponible en: http://www.efdeportes.com/efd108/obse rvacion-de-las-acciones-ofensivas-enun-equipo-de-futbol.htm.

85. Taylor, JB, Mellalieu, SD, James, N, Barter, P. Situation variable effects and tactical performance in professional association football. International Journal of Performance Analysis in Sport 2010;10(3): 255-270.

86. Tenga, A., Ronglan, L.T. y Bahr, R. Measuring the effectiveness of offensive match-play

professional soccer. European Journal of Sport Science 2010;10(4):269.

87. Trninié, S., Milanovic, D. y Dizdar, D. En qué se diferencian los ganadores y los perdedores en baloncesto. INFOCOES, II. 1997; (1), 56-65.

88. Valez, A., Areces, A., Blanco, H., Arce, C. Diseño y aplicación de una batería multidimensional de indicadores de rendimiento para evaluar la prestación competitiva en el fútbol de alto nivel. International Journal of Sport Science 2011; VII(23):103-112.

89. Voyer, D., Kinch, S. y Wright E.F. The home disadvantage: examination of the self-image redefinition hypothesis. Journal of Sport Behavior 2006;29:270279

90. Zubillaga, A. La actividad del jugador de fútbol en alta competición: análisis de variabilidad.[Tesis Doctoral] Universidad de Málaga; 2006.

Rev. Ib. Cᄃ. Act.Fis. Dep. 